\title{
This Flow Ain't Free: Generative Elements in Kendrick Lamar's To Pimp a Butterfly
}

\author{
John J. Mattessich
}

NOTE: The examples for the (text-only) PDF version of this item are available online at: https://www.mtosmt.org/issues/mto.19.25.1/mto.19.25.1.mattessich.php

KEYWORDS: hip hop, flow, Kendrick Lamar, popular music, semiotics, cultural studies

ABSTRACT: This article presents analyses of three songs from To Pimp a Butterfly with respect to the relationship between Lamar's flow and the instrumental track in each song. A spectrum of flow is proposed, which consists of flow that is at one extreme derivative (metrically and hypermetrically aligned with the instrumental track) and at the other generative (flow and instrumental track are discontinuous). This framework is then considered with respect to normative stylistic considerations and used as a means of interpretation in tandem with the lyrics.

DOI: $10.30535 / \mathrm{mto} .25 .1 .11$

Received May 2018

Volume 25, Number 1, March 2019

Copyright $\odot 2019$ Society for Music Theory

[1] In his article "On the Metrical Techniques of Flow in Rap Music," Kyle Adams defined flow in rap music as "all of the rhythmical and articulative features of a rapper's delivery of the lyrics" (Adams 2009, [6]). He outlines how delivery can affect the expressive quality of the song, discussing a variety of rhythmic techniques that include the placement of accented syllables in relation to the beat, as well as the variance of note length and subdivisions of the beat. He summarizes the defining characteristics of flow into two main categories: metrical techniques and articulative techniques. Metrical techniques have to do with the placement of rhyming and/or accented syllables, the number of syllables in a beat, and the degree of correspondence between syntactic units and measures. Articulative techniques deal with the amount of legato or staccato used, placement of syllable onset relative to the beat, and the hard or soft articulation of consonances. While a rapper might not strategically manipulate all of these factors at the same time, the breakdown into characteristic elements provides a useful heuristic for the analyst.

[2] Kendrick Lamar's To Pimp a Butterfly (2015) contains a wide range of examples that are relevant to Adams's notion of flow. An analysis of Lamar's music that uses that conceptual framework reveals that the rhythmic quality of his flows lies on a spectrum whose extremes range from flows that reinforce every downbeat and subdivision to flows on the other end that reinforce neither a downbeat nor a subdivision. Between these two extremes lies a variety of rhythmic ambiguities that can serve to reinforce, disrupt, or provide rhythmic counterpoint to the established meter, the extent to which varies from song to song. Flow with the quality of having derived its metrical 
structure from that of the instrumental track will be called derivative. Flow that functions independently of the instrumental track (generating its own structure and internal rhythmic relationships) I will call generative. Robert Walser describes the relationship between a rapper and the instrumental track of a hip hop song: "The music is not an accompaniment to textual delivery; rather, voice and instrumental tracks are placed in a more dynamic relationship in hip hop, as the rapper interacts with the rest of the music" $(1995,204)$. Adams (2008) adopts a similar framework regarding delivery of text and the instrumental track. Along with his analytical categories, this provides a foundation for the discussion of flow through the lens of vocal rhythm and its relationship to the rhythm and meter established by the instrumental track. The following three analyses of tracks from To Pimp A Butterfly reveal a dynamic relationship between flow and instrumental track and posit an interpretation that accounts for issues of musical meaning that arise owing to that relationship. In my analysis of "King Kunta," I provide an example of flow that is almost entirely derivative of this underlying structure. My analysis of "Momma" provides an example of a flow that is hypermetrically aligned with the instrumental track, but the flow eschews the implied subdivisions of that structure. Its "alignment with ambiguities" displays characteristics of a derivative flow with generative rhythmic elements. In my final analysis, I show how "For Free? - Interlude" exemplifies a flow that is entirely independent of the metrical structure of the instrumental track. Following these analyses, a sample interpretation will demonstrate how these relationships between flow and instrumental track function in a larger discourse, and how they might be brought into dialogue with existing cultural literature.

[3] A growing number of analysts have proposed models dealing with flow in rap music, including Robert Walser (1995), Oliver Kautny (2015), Kyle Adams (2008, 2009), and Adam Krims (2000), among others. Building on the ideas proposed by these scholars, the following analyses approach the music of Kendrick Lamar by focusing on how different types of flow are manifested, emphasizing the relationship between the metrical implications of the rapper and the instrumental track. Finally, I consider what discourses arise with the identification of these elements of flow.

[4] According to Walser, the prevailing compositional paradigm in hip hop situates the rapper and the instrumental track in a dynamic relationship, but one in which the rapper interacts in a directed manner with the groove:

Because the groove itself is non-teleological, it situates the listener in a complex present, one containing enough energy and richness that progress seems moot. Form and direction are imposed on the song by the rapper through rhetorical fiat, by means of rhythmic patterns, rhymes schemes, the ideas and exhortations of the lyrics, and the verse/chorus alternation. ${ }^{(1)}$ (Walser 1995, 204)

His model posits the instrumental track as a primary musical resource for the rapper, while the vocals serve to elucidate innate musical possibilities. Although structure is thus delineated by the vocals, it is ultimately derivative of and reliant upon the instrumental track; flow imposes its teleology onto the instrumental track, but it can only do so by being subjected to the musical characteristics inherent to the beat itself and by manifesting itself through the expression of those characteristics. The rhythmic patterns, rhyme schemes, and formal considerations that Walser mentions are all expressive only to the extent that they exist within this framework. ${ }^{(2)}$ He identifies a material space that creates the environment with which rappers interact and deploy their own musical and cultural agency. Lamar creates interest through the interaction of musical ideas, and the ways in which his deployment of musical ideas relate to or contrast with the instrumental track provides a spark for interpretation.

\section{Derivative Flow - "King Kunta"}

[5] An example derivative flow can be found in the chorus of "King Kunta." The structure of his flow over a standard ${ }_{4}^{4}$ meter privileges downbeats with dynamically stressed syllables and sixteenth-note subdivisions that reinforce the metrical hierarchy. Additionally, a traditional backbeat conveys the metrical division in each measure, identifying 2 and 4 as accented beats. A four-bar hypermeter is established as the chorus lasts for four repetitions of four distinctively 
stressed downbeats equally subdivided into four sixteenth-notes. The rhyme scheme reinforces this crystalline regularity with its centricity on the fourth downbeat of each line. Example 1 shows

these relationships in a flow diagram with verbally accented syllables (by way of an increase in volume) bolded and rhyme scheme highlighted. ${ }^{(3)}$

[6] The third verse of "King Kunta" maintains much of the chorus's structural regularity, but the flow employed by Lamar differentiates it in two distinct ways. As seen in Example 2, the rhyme scheme becomes couplet-based rather than lasting for four bars, and the lyrics become more densely packed, as Lamar fills the subdivisions between downbeats with a rapid-fire delivery. Lamar's regularity of subdivision and rhyme scheme serves to maintain the rhythmic and metrical structures established earlier in the song. These two examples illustrate how the rhythmic structure of flow can be derived from the structure of the instrumental track. In these cases, the articulative and metrical elements of Lamar's delivery coincide with the typical metric implications of a 4 meter, with respect to appropriately stressed downbeats and subdivisions. Bolded in Example 2 are accented syllables that reflect a rise in pitch, while the highlighting once again reflects the rhyme scheme, this time in couplets. Bolded syllables typically fall on prominent downbeats 1 and 3, while off-beat accents appear as eighth-note anacruses to 1 or 3 (in both cases, the first syllable of "everybody"). In this verse, the rise in pitch on the accented syllables reinforces the ${ }_{4}^{4}$ meter of the instrumental track, while the couplet rhyme scheme differentiates the verse from the chorus. The instrumentation behind Lamar, which had been building progressively throughout the song from a single bass line and drum set to a full texture with multiple synths and vocal samples, is drastically reduced in verse 3 . The backbeat is removed; only a guitar and bass line remain. The absence of the drums creates a temporary metrical void, highlighting Lamar's faster delivery in this section. Even without the drum pattern to align our hearing of the meter, Lamar's flow fills in nearly every subdivision, which allows a shift in timbre and texture without jeopardizing metrical integrity.

\section{Derivative Flow with Generative Characteristics - "Momma"}

[7] The relationship between instrumental track and flow is not always so clear. Much of the time there are certain rhythmic elements of a rapper's flow that are discontinuous with the metrical implications of the instrumental track. Composition of the beat can also obscure the meter in such a way that the rapper must generate elements of a metrical structure and/or other rhythmic relationships within the flow itself. In these instances, the musical characteristics of the instrumental track that establish order are masked but still in control; the flow (if it is to align with the beat) manifests the metrical irregularity to which it is subject through a grouping structure that is out-of-phase with the underlying metrical structure. In "Momma," the instrumental track contains a similar kick/snare pattern that affirms the same ${ }_{4}^{4}$ metrical hierarchy that was found in "King Kunta," but there are cowbell hits on the sixteenth-note preceding the accented beats that anticipate them, which gives the drum pattern a certain unstable quality - the quality of tripping forward into each accented beat. Lamar's flow employs a rhyme scheme with an irregular internal rhyme and a regular end rhyme. Additionally, the enjambment of syllables in order to create a point of landing at the end of each line (around beat 4) serves as a point of coherence between the otherwise largely disjunct flow and instrumental track. More succinctly, the flow and instrumental track are unified at the level of the hyperbeat, but not at the level of the beat. The rhyme scheme falling consistently on or around beat four confirms that the flow and the instrumental track are inexorably linked. Considering this structural linkage, enjambment and rhythmic irregularity in the vocals act as generative elements (that is to say, they partially obscure the metrical hierarchy) by masking the feeling of subdivisional alignment that one would find in a strictly derivative flow, such as "King Kunta."

[8] The organization of Example 3 is based on Adams's method of displaying flow used in Example 2 , which identifies the location of syllables with respect to beats. The diagram is modified from his formulation: instead of using a metrical grid that implies strict subdivisions, beats are represented as equidistant points in time. The subdivisions have been eliminated to show the flexibility with which Lamar's flow operates. His lines tend to land around beat 4 , as can be seen in the rhyme scheme, but the alterations he makes are quite loose compared to the rigid beat 4 rhyme scheme 
found in "King Kunta." This flow demonstrates a reliance upon the metrical and rhythmic foundation of the instrumental track fitting in with Walser's compositional model, but the treatment of individual beats is flexible; they become points of reference, rather than landing points as Lamar gracefully weaves unencumbered around the structure they project. The diagram reflects this looseness as word placement either on or around each downbeat indicates its placement relative to the groove. Although the accents of the speech patterns he uses create discontinuity with the instrumental track, the rhyme scheme and the tendency to follow downbeat guide points (not always strictly) reinforce a hypermetric regularity in both the vocals and the instrumental track. In this way, generative elements in Lamar's flow manifest as nebulous subdivisions of the meter, which is still clearly articulated at a higher level.

\section{Generative Flow - "For Free?"}

[9] Pushing this relationship to a breaking point, "For Free?" contains a flow and instrumental track that are completely stratified (there is no structural relationship between the rhythms in the vocals and the metrical implications of the instrumental track). The vocal line is entirely selfgenerative with respect to its structure and motivic content. Lamar creates an independent structure and develops that structure using only the sounds of the words in the lyrics, as well as his accents and the cadence in his delivery. The instrumental track is comprised of a jazz combo with multiple players improvising at the same time. Lamar's flow is reminiscent of a spoken word performance, as his segmentations are guided more by semantic concerns and affect than metrical concerns. This self-generative nature differs significantly from the standard text-music relationship in hip hop, moving away from Walser's model, which privileges a strict adherence (at a basic structural level) of instrumental track and flow to the same meter (although it maintains its interactive nature, in a slightly different guise than the past two examples, which will be explored later on).

[10] The process that went into recording "For Free?" also requires some explication, as it varies significantly from the previous two examples. Piano player Robert Glasper describes an interaction with producer Terrace Martin in an interview: “I asked, 'What kind of vibe do you want?' Terrace was like: 'Straight up, Kenny Kirkland, Branford [Marsalis], late '80s.' And I knew exactly what he was talking about" (Deshpande 2015). Not only was the song recorded with live musicians sitting in on the session, but Glasper also states that he went back at Lamar's request after the song had been completed and overdubbed the series of chord hits that comes in Section 6. While one might consider the stylistic allusion to late-80s jazz as a sample of sorts, the interaction of Lamar and the instrumental musicians is much more along the lines of a musician playing with a jazz combo.

Glasper reflects on this dynamic from the sessions spent on the album later on in the interview: "I was actually thinking of it like he was a saxophone player, you know what I mean. Not like a singer or an $\mathrm{MC}$, but literally like a saxophone player, it was like some jazz shit. So it was dope when he said 'Hey, do this'”' (Deshpande 2015).

\section{A Word on Notation (Interlude)}

[11] Hip hop music relies primarily on aural transmission. As such, it is generally not notated using Western European conventional notation and thus graphic representation in analysis becomes an explicitly interpretive act. I owe a debt to a number of scholars who have created novel ways of representing rap music on the page: Manabe (2019), Ohriner (2016) (both contained in this issue), Adams (2009, 2015), Kautny (2015), and Krims (2000), among others. The two preceding analyses have highlighted that, while the stylistic expectation in hip hop suggests a metrical grid (as in Examples 1 and 2) that implies a direct rhythmic and metrical correlation between flow and instrumental track, deviations can occur that obscure that relationship. Both "King Kunta" and "Momma" contain flows in a derivative relationship to the beat, although the latter contains a greater amount of discontinuity in its rhythmic relationships (while maintaining the expected hypermetric considerations). Revisiting the notion of a derivative-generative spectrum, one might imagine a crystalline metrical grid dissolving as one moves from derivative to generative. This 
movement away from notation with generative flow is not one of abstraction, but one that allows the music to speak on its own terms, and for us to meaningfully engage in a way that is primarily determined by the music. While the imposition of an analytical framework such as metrical determination is helpful with music containing a regular meter, movement away from this metrical regularity necessitates a movement away from the strict rhythmic grid that characterized the analyses of "King Kunta" and "Momma." The dissolution of the metric grid from Example 1 to Example 3 is as much an interpretive decision as it is a performative one. It is meant to display a mode of listening in which elements of meter slowly decrease in importance as the listener begins to focus on the independent structures of the flow and the instrumental track (and the separate characteristics that determine those structures), rather than on their alignment. The following example ("For Free?") consists of flow that is entirely generative; while a process of close-reading and skillful manipulation of rhythmic notation might accurately record the precise relationship between the vocals and the instrumentals, I find these relationships to be well beyond the ear of the average listener (although there are certain interactions made explicit by the artists involved, which will be addressed). It may be possible to represent any series of durations in standard Western classical notation, but one must also consider what is being said about the music in the act of transcription; the imposition of a metrical structure in the manner of the first two examples would say more about the ill fit of that analytical framework than it would about the musical cohesion of

"For Free?." I am not compelled to make the argument that Lamar is capable of creating incredibly complex flows that demonstrate knowledge and mastery of the genre; that element of the music speaks for itself. Instead, I suggest a means by which listeners might pay attention to how he varies the relationship between the instrumental track and the elements of his flow that create a sense of cohesion and unity. For this reason, I find it best to approach this music aurally, and forego notated examples in favor of audio.

\section{Generative Flow (cont.) - "For Free?"}

[12] The rhythmic organization of the vocals operates on local levels (small groupings of words), and large-scale levels that demonstrate musical development across sections. The segmentation of the song's six sections is based on the recurrence of a refrain text ("This dick ain't free"), and the framework for delineating subsections within each section is based on the continuously developing usage of similar articulative and metrical flow techniques. Focusing specifically on sections 1, 3, and 6 reveals an organizational framework that arises organically and develops continuously throughout the course of the song. In this analysis, the lyrics will be laid out in a way that corresponds to these three-section divisions. Each section is delineated by phrasing or rhyme scheme, rather than an alignment with the meter of the instrumental track.

You lookin' at me like it ain't a receipt,

Like I never made ends meet,

Eating your leftovers and raw meat.

[13] Section 1 (Audio Example 4) is the shortest of the six, consisting of three lines. Despite its brevity, it sets up a structural paradigm and thematic tendencies that will recur within subsequent sections. Foremost, the rhyming syllables in each line serve to delineate phrasing and cadence in lieu of being guided by the rhythm of the instrumental track. Lamar emphasizes the rhyme of "receipt," "meet," and "meat" to segment the section into three distinctive lines, the last of which gradually slows down his rapid-fire delivery in order to transition back into the refrain. This slowdown at the end of the section which helps articulate the three-part subsectional division will become structurally important as the song continues.

I mean, baby, you really think we could make a baby named Mercedes without a Mercedes Benz and twenty-four inch rims, five percent tint, and air conditioning vents?

Hell fucking no. 
[14] Section 3 (Audio Example 5) continues the development of strategic elements arising in Section 1. The section is split up into three subsections, the first of which serves as an initial contrasting subsection, whose longer rhythmic values help to accentuate the quickly changing rhythms that follow in the second subsection. The rapid-fire rhythms in the second line are juxtaposed with a rhyme scheme that plays off of "baby" in the first subsection. The rhyme scheme then morphs into a non-regular rhyme scheme (4-6-4-7 syllable count into each rhymed syllable) at the word "Benz," as is shown by the bolded words in Example 5. Although these rhymes do not follow a regular pattern, they do serve as accents within his flow that create a grouping structure independent of any meter in the instrumental track. Finally, the last line serves as the closing subsection ("Hell fucking no"), as in the past two sections; Lamar's delivery slows down in preparation for a restatement of the refrain.

Matter o' fact it need interest, matter o' fact it's nine inches.

Matter o' fact see our friendship based on business, pension, more pension, you're pinchin',

my consensus been relentless, fuck forgiveness, fuck your feelings.

Fuck your sources, all distortion, if we fuck it's more abortion, more divorce

courts and portion my check with less endorsement left me dormant, dusted, doomed, disgusted, forced with fuck you think is in more shit?

Porcelain pipes pressure, bust 'em twice, choice is devastated, decapitated the horseman.

Oh, America you bad bitch, I picked cotton that made you rich Now my dick ain't free

[15] Section 6 (Audio Example 6) serves as the teleological climax of the song, as all of the developing musical and rhythmic elements throughout the previous sections reach a peak of intensity. Its opening, no longer a brief subsection, contains a statement beginning "matter o' fact," followed by a varied repetition of the statement, and finally a continuation that devolves into a strong compound metrical implication. This process effectively merges the first and second subsections with respect to their structural roles in previous sections, as the continuation of the first section becomes the impetus that drives the second section. The internal rhyme scheme, revolving mainly around triplets, grows in intensity through a confluence of factors including literary consonance, repetition of pitch and rhythm, and the articulation of consonants. Additionally, the accompaniment grows in intensity, complementing the climactic flow with a series of hits that reinforces the vocal accents that Lamar employs. This interaction serves as the only noticeable rhythmic relationship in the whole song; the significance of this interaction will be discussed later in relation to the stylistic ramifications of the song. A noticeable break in the pattern ("Oh, America you bad bitch") signifies the closing subsection and the end of the song. The climactic intensity mounting throughout the song and coming to the fore in section six dissipates with this final subsection, and the refrain text is heard one more time.

[16] Recalling the common organizational framework continuously developing between sections, each section follows a tripartite model that increases in complexity as the song goes on. The first part of this three-subsection partitioning establishes a motivic idea or precedent that will be developed within the whole section. The middle subsection then develops or contrasts this motive from the opening. In the closing subsection, the note lengths increase as the section prepares for the return to the refrain. Usually this will be some kind of closing or cadential material for the section, either in terms of musical material or in terms of subject matter. These subsections change in their complexity and role, yet their presence throughout creates a thematic continuity in each section and allows for an analysis of the work based solely on how text and music are shaped by articulative and metrical choices in the vocals.

\section{Flow and Stratification}

[17] From a rhythmic standpoint, generative flow and the instrumental track are entirely stratified in "For Free?," as they do not align structurally at any given point. A lack of structural cohesion does not necessarily mean that the two independent musical streams never cross paths, as there are 
points in the song when the rhythm section responds to imagery (snare cross-stick imitating a knock at "had the door knocking") or consistent rhythmic accents in the flow (pseudo-metrical implication in section 6). These temporary points of cohesion are subtle, but the implications that go along with the rhythm section's responses to the text are extremely profound; these interactions invert Walser's hip hop compositional paradigm by eliciting a response from the accompaniment to structural and thematic cues in the flow, rather than the rapper responding to structural cues in the accompaniment. As Glasper discussed in the interview cited earlier, the musicians involved in the recording process played off of one another in a form of musical discourse suggestive of a jazz combo.

[18] Although these cues that the rhythm section picks up near the end of "For Free?" do suggest a temporary alliance of rhythmic agencies, they do little to ground the song structurally. Temporary moments of thematic cohesion point towards structural parallels, but these points do not create large-scale cohesion between the largely independent musical streams (instrumental track and vocals). Obviously, these streams are presented simultaneously, but mashing these disparate elements together is more than musical miscellany. John Covach (2001) describes this notion of textural stratification as "the idea that musical passages may be made up of layers of musical activity that, while heard together, resist reduction down to a single musical event." His discussion specifies that the level of coordination among strata in a work determines whether or not we as listeners can hear the work "harmonically," which refers to the interactivity of simultaneities among strata. In the context of meter, a "rhythmic" interpretation of the work could search for metrical similarities between the instrumental track and the flow, which (other than the temporary points of cohesion discussed above) simply do not occur. As Covach explains, when the strata distinguish themselves without fusing to one another, or when reduction to a single event is impossible, they are "loosely coordinated" as opposed to highly coordinated, lending themselves more readily to reduction. In the case of meter in "For Free?," loose coordination exists between the vocals and instrumental track, as the two rarely coincide.

[19] Analysis of loosely coordinated strata requires modeling that does not encourage what Covach calls "harmonic" (adapted for this analysis as "metric") listening. In this particular case, the rhythmic interaction between the musical layers is coloristic rather than structural; the organization and unification of the strata operates at a level that supersedes that of meter. To this end, the rhythm section and the generative flow enter not a rhythmic discourse, but a semiotic one.

[20] The dynamic relationship of Lamar's flow with the instrumental track on these selections from Butterfly can be qualified within two categories. Flow that is derivative relies upon the metrical and hypermetrical considerations of the instrumental track in order to determine its overall structure. Derivative flow may contain elements that create an ambiguous relationship between flow and instrumental track, but ultimately there is a fundamental structural alignment between the two entities. The primary musical material within a generative flow is derived from the sound and cadence of the words, not from the structural hallmarks of the instrumental track itself. Because the stylistic nature of hip hop lends itself so readily to derivative flow (which is to say that we as listeners tend to expect rappers to rap in relation to the instrumental track), the stratified texture in "For Free?" is particularly unusual. By definition, flows that are fundamentally derivative can contain generative elements while retaining metrical and/or hypermetrical alignment with the instrumental track, but flows that are fundamentally generative cannot contain derivative elements. This specificity of meaning aligns with Robert Hatten's (1994) application of markedness theory in musical expression.

[21] Hatten's (1994) application of markedness evaluates all forms of difference as oppositional: an asymmetrical relationship between two entities determines the "marked" quality of one and the "unmarked" quality of the other. This asymmetry necessarily creates a narrower range of meaning for the marked entity, and results in the entity's less frequent appearance within stylistically expressive contexts. Markedness originates when a unique or salient event arises, which the competent listener understands as creating or implementing a new opposition (36). For example, most hip hop songs fall into a standard ${ }_{4}^{4}$ meter; thus a song in ${ }_{4}^{3}$ exemplifies an entity that is stylistically marked. As ${ }_{4}^{4}$ is stylistically normative, the enculturated hip hop listener has an 
expectation that most songs will be in ${ }_{4}^{4}$; a song in ${ }_{4}^{3}$ draws attention to the fact that it is both in a triple meter (it is marked) and not in quadruple meter (it is not unmarked).

[22] In hip hop, generative flow is marked with respect to derivative flow. Derivative flow is understood to be stylistically normative, so the incorporation of generative elements introduces an oppositional relation to the paradigm. This stylistic competency then determines that an entire song normalizing the paradigm of a generative flow as structural is viewed as a "marked event." While rapping just off the beat might sound hip in a certain context, rapping completely independent of any sense of meter (especially while a rhythm section is laying down a groove!) is certainly enigmatic. The markedness of generative flow creates a specificity of musical meaning that requires a more robust analysis in order to understand fully; it impels us to dive into the semantics of the text.

\section{Interpreting Flow}

[23] Bringing the generative flow to a reading of "For Free?" as a text can inform an interpretation beyond merely stating that "generative flow sounds weird." A focus on the dynamic of the rapper, the instrumental track, and the relationship between the two might suggest a politics of art, or help uncover a musical subjectivity present in the work. My musical analysis has revealed a structure that builds throughout the song to a climax in the final section, which is predicated on the disjunction of flow and instrumental track. Now, we turn to the piercing final line of the song: "Oh America you bad bitch, I picked cotton that made you rich, now my dick ain't free." We are presented with a metaphor, America as a "bad bitch," as well as with plantation imagery. There are issues of gender, as well as race and domination that come to the fore in this line, and while their context is a particularly charged one, it is one that warrants discussion. The musical disjunctures as well as the forceful lyricism gesture toward a more complex reading of the song.

[24] First, we begin by examining the refrain text: "this dick ain't free." In the context of the song, it could have two meanings. The first meaning arises in the context of the argument that opens the song. In a lover's spat, a woman's voice insults Lamar (or rather, the character who is being formed within the concept album), lambasting him for not spending enough money on her and for not having enough clout to deserve her affections. The rest of the song serves as his response to her assertion; he identifies the sacrifices he made, as well as his own animal-like subjugation ("eating your leftovers and raw meat"). As the song goes on, the references Lamar makes grow in scope: American capitalism ("Livin' in captivity raised my cap salary/Celery, tellin' me green is all I need"); a Mercedes Benz; and an allusion to "forty acres and a mule," which functions as a synecdochic reference to the ways in which the American nation-state has consistently and systematically throughout its history antagonized those who seek Black liberation and selfdetermination. The first reading of "this dick ain't free" functions within the context of the lover's spat; we hear Lamar declare his independence and assert his own self-worth, as if he is making it clear that he is no longer going to engage in a sexual relationship with someone who does not value him as a person. By the end of the song, we begin to get an idea that "this dick ain't free" functions in a slightly different way; while he may assert his independence and self-worth, the fact remains that his relationship to America is "based on business," and that "if we fuck it's more abortion/more divorce courts and portion my check with less endorsements," a less-than-ideal situation. Caught in the exploitative snares of American capitalism, we begin to understand that "I picked cotton that made you rich/Now my dick ain't free" functions not so much as an assertion of independence, but as a presentation of fact: he is not free because he remains subjugated by oppressive systemic racism.

[25] Understanding these dual meanings and the social implications of "America, you bad bitch" means placing these utterances within a broader social context. Imani Perry identifies a crisis of black male subjectivity, which asserts itself problematically over the bodies of women as something that occurs within the genre of hip hop: "It is, in fact, a sense of powerlessness in the face of white masculinity, and the fear of being pimped at the hands of wealthy white recording moguls, that guides the hypermasculinist moment, and the heterosexist moment as racial anxiety is articulated 
through a patriarchal cultural lens when the fear of being "bitched" finds artistic expression" (2004, 124). Perry identifies a tendency within American culture that devalues the black subject and whose white supremacist consumption of the black male body constitutes a gender-based oppression. "Discussions of black male patriarchy or sexism should therefore always first consider the intersectionality of blackness and masculinity, as these discussions may otherwise easily fall into determinative pejoratives building on the gendered oppression of black men" (120). Tricia Rose also identifies this type of social anxiety, which manifests as sexism in Black Noise: Rap Music and Black Culture in Contemporary America: "Some of this hostility toward women is related to the dominant cultural formula that equates male economic stability and one's capacity to be a family breadwinner with masculinity" $(1994,171)$. The politics and social forces that a rapper like Lamar faces (both in the context of the album, and in real life) are complex, considering the fact that the genre of hip hop, which has its roots in the urban Black experience, has been co-opted by white industry executives, commodified, and sold to a predominantly white audience. With the continuous exclamation "this dick ain't free," Lamar's character in the album embodies the anxiety identified by Perry and Rose as the result of generations of oppression and dehumanization. His subjection to the white supremacist gaze of American society makes it so that even his own cultural production is co-opted by what bell hooks identifies as "white supremacist capitalist patriarchy" (1994, 143).

[26] That which is "American" is inherently grounded in a white supremacist social and cultural framework. But what characterizes Lamar's output is not merely an opposition between being Black and being American. Following Stuart Hall (1992), Lamar embodies a complex subjectivity within the hip hop tradition that crosses the cultural boundaries of a diasporic Black cultural repertory and a hegemonic American socio-cultural discourse. Hall posits his formulation of Black popular cultures as the site of contestation, a logic of coupling rather than of binary opposition: "You can be black and British, not only because that is a necessary position to take . . but because even those two terms, joined now by the coupler 'and' instead of opposed to one another, do not exhaust all of our identities" $(1992,29)$.

[27] Wrapped up in this diasporic multiplicity of identities is the poetics of address known as Signifyin(g). Cheryl Keyes identifies this as a particularly important part of production and performance within the hip hop tradition, and she quotes Claudia Mitchell-Kernan, whose seminal work on signifyin' identifies that:

“. . . the Black concept of signifying incorporates essentially a folk notion that dictionary entries for words are not always sufficient for interpreting meanings or messages, or that meaning goes beyond such interpretations. Complimentary remarks may be delivered in a left-handed fashion. A particular utterance may be an insult in one context and not another. What pretends to be informative may intend to be persuasive. The hearer is thus constrained to attend to all potential meaning-carrying symbolic systems in speech events - the total universe" (Keyes 2002, 131-32).

Within the context of signifyin', a rapper can reach into the depths of a performative tradition that plays with meaning: things are as they are, and simultaneously other than they appear to be.

[28] This is the space that is opened by the disjunction of generative flow in "For Free?." There comes a point where one realizes that what can be said is vastly eclipsed by that which is necessarily unsaid, yet deeply felt and recognized. A generative flow might create its own structure by a similar process to that by which a derivative flow's crystalline meter embodies a specific order and logic. These mere categorizations are woefully inadequate at describing what is really going on; the more we seek to essentialize these ephemeral performativities, the further we get from uncovering the vast network of cultures and meanings that are manifest in these instances. Paul Gilroy, in The Black Atlantic, describes what he calls a politics of fulfilment as a "counterculture of modernity": "The politics of fulfilment. . .creates a medium in which demands for goals like nonracialised justice and rational organization of the productive processes can be expressed. It is immanent within modernity and is no less a valuable element of modernity's counter-discourse for being consistently ignored" $(1993,37)$. These are a politics which can come to be known through discourse, those that can be named and grappled with - the white supremacist gaze, the capitalist 
mode of production; it is a material, referable form of identity. He also identifies what he calls a "politics of transfiguration": "[Politics of transfiguration] points specifically to the formation of a community of needs and solidarity which is magically made audible in the music itself and palpable in the social relations of its cultural utility and reproduction. . . This politics exists on a lower frequency where it is played, danced, and acted, as well as sung and sung about, because words, even words stretched by melisma and supplemented or mutated by the screams which still index the conspicuous power of the slave sublime, will never be enough to communicate its unsayable claims to truth" $(1993,37)$. Moving away from the semiotic and the language of oppositions, Gilroy identifies both of these modes of political engagement as part of a Black Atlantic counterculture of modernity. Gilroy's notions of politics speak to that which is simultaneously material and ineffable, to a mode of discourse that seeks to uncover what cannot be uncovered, and that finds restitution in the sublime. It is this sublimation towards which my analytical lens gestures, and in its act of gesturing falls necessarily short. In Kendrick Lamar's flow, in the interaction of disparate musical elements, we might catch just a gleam of these performed politics. We might do well, following Perry, "to use the creativity and ideology contained within the music to enrich the ways we think about society and the ways we create contemporary theory" $(2004,101)$. That in analyzing hip hop, we might understand not just the radical potential of the marginalized subject, but also a profound politics of the everyday coexisting with one another. We must not be content with the socialized binaries that seek to categorize and alienate, but understand that forms of identity arise within the ruptures of hegemony. In hip hop, we see the fusion of high and low, margins and center, old and new; Lamar performs not within the context of these binaries, but above, below, and through them, unencumbered by their arbitrary closures (Hall 1996, 117). His performance is a testament not just to the potential of hip hop to speak profound truths to power, but also to say what cannot be said - what must be lived, felt, seen, and heard in the politics of everyday life. ${ }^{(4)}$

John J. Mattessich

Indiana University - Bloomington

Jacobs School of Music

1201 East Third Street

Bloomington, IN 47405

jomatt@iu.edu

\section{Works Cited}

Adams, Kyle. 2008. "Aspects of the Music/Text Relationship in Rap." Music Theory Online 14 (2). http://www.mtosmt.org/issues/mto.08.14.2/mto.08.14.2.adams.html.

2009. "On the Metrical Techniques of Flow in Rap Music." Music Theory Online 15 (5). http://www.mtosmt.org/issues/mto.09.15.5/mto.09.15.5.adams.html.

2015. “The Musical Analysis of Hip-Hop." In The Cambridge Companion to Hip-Hop, ed. Justin Williams, 118-34. Cambridge University Press.

Covach, John. 2001. "Some Remarks on Textural Stratification in 20th-Century Music." Lecture, Eastman School of Music, Rochester, NY.

Deshpande, Jay. 2015. “Jazz Pianist Robert Glasper on His Role in Kendrick Lamar's To Pimp a Butterfly." Slate.com, March 27.

http://www.slate.com/blogs/browbeat/2015/03/27/kendrick_lamar_s_to_pimp_a_butterfly_robert_glasper_on_what.

Gilroy, Paul. 1993. The Black Atlantic: Modernity and Double-Consciousness. Harvard University Press.

Hatten, Robert. 1994. Musical Meaning in Beethoven: Markedness, Correlation, and Interpretation. Indiana University Press.

Hall, Stuart. 1992. "What Is This 'Black' in Black Popular Culture?” In Black Popular Culture: A Project by Michele Wallace, ed. Gina Dent, 21-33. Bay Press. 
1996. “Minimal Selves." In Black British Cultural Studies, ed. Houston A. Baker, Jr., Manthia Diawara, and Ruth H. Lindeborg, 114-19. University of Chicago Press.

hooks, bell. 1994. Outlaw Culture. Routledge.

Kautny, Oliver. 2015. "Lyrics and Flow in Rap Music." In The Cambridge Companion to Hip-Hop, ed. Justin Williams, 101-17. Cambridge University Press.

Keyes, Cheryl. 2002. Rap Music and Street Consciousness. University of Illinois Press.

Krims, Adam. 2000. Rap Music and the Poetics of Identity. Cambridge University Press.

Manabe, Noriko. 2019. “Chants of the Resistance: Flow, Repetition, Inclusivity.” Music and Politics 13 (1). https://quod.lib.umich.edu/m/mp.

Ohriner, Mitchell. 2016. “Quantizability in 'Momma,' Verse Two." Paper presented at the Society for Music Theory Popular Music Interest Group Meeting. Vancouver, Canada.

Perry, Imani. 2004. Prophets of the Hood: Politics and Poetics in Hip Hop. Duke University Press.

Rose, Tricia. 1994. Black Noise: Rap Music and Black Culture in Contemporary America. University Press of New England.

Walser, Robert. 1995. "Rhythm, Rhyme, and Rhetoric in the Music of Public Enemy." Ethnomusicology 39 (2): 193-217.

\section{Footnotes}

1. It is also important to note that Walser's (1995) compositional paradigm deals primarily with sample-based hip hop being produced by members of the Bomb Squad. Although To Pimp a Butterfly does not rely as heavily on samples as Walser's source material, the prototypical modern hip hop beat does typically rely on a looped musical fragment rather than being throughcomposed. While the difference between a looped sample and a looped composed fragment is one of production techniques, the standard function of both is roughly equivalent.

Return to text

2. It should be noted here that while analyzing the relationship of flow to the instrumental track assumes the instrumental track as a precursor to the rapper's vocals, this is not always the case. Frequently, producers and rappers will collaborate simultaneously on tracks in order to create a dynamic interplay between the instrumental music and the vocals (as will be seen later in "For Free?").

Return to text

3. Adams 2009 serves as the model for this style of notation.

Return to text

4. This article is an expanded version of a talk given as part of an analytic roundtable discussion at the 2016 meeting of the Society for Music Theory's Popular Music Interest Group. Special thanks are due to the other participants of that discussion, whose work is represented in this symposium. Additionally, I am incredibly grateful to Kyle Adams, Jane Clendinning, Shane Vogel, and the many inspiring professors and colleagues whose insights and efforts were essential in bringing this article to completion.

Return to text

\section{Copyright Statement}

Copyright (C) 2019 by the Society for Music Theory. All rights reserved.

[1] Copyrights for individual items published in Music Theory Online (MTO) are held by their authors. Items appearing in MTO may be saved and stored in electronic or paper form, and may be shared among individuals for purposes of scholarly 
research or discussion, but may not be republished in any form, electronic or print, without prior, written permission from the author(s), and advance notification of the editors of MTO.

[2] Any redistributed form of items published in MTO must include the following information in a form appropriate to the medium in which the items are to appear:

This item appeared in Music Theory Online in [VOLUME \#, ISSUE \#] on [DAY/MONTH/YEAR]. It was authored by [FULL NAME, EMAIL ADDRESS], with whose written permission it is reprinted here.

[3] Libraries may archive issues of MTO in electronic or paper form for public access so long as each issue is stored in its entirety, and no access fee is charged. Exceptions to these requirements must be approved in writing by the editors of $M T O$, who will act in accordance with the decisions of the Society for Music Theory.

This document and all portions thereof are protected by U.S. and international copyright laws. Material contained herein may be copied and/or distributed for research purposes only.

Prepared by Andrew Eason, Editorial Assistant

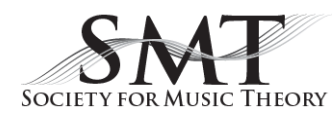

